\title{
Caracterización de los niveles de razonamiento de Van Hiele específicos a los procesos de descripción, definición y demostración en el aprendizaje de las razones trigonométricas ${ }^{1}$
}

\author{
Characterization of levels of van Hiele reasoning processes specific \\ description, definition and demonstration in learning trigonometric ratios
}

Caracterização dos níveis de van Hiele raciocínio processa descrição específica, a definição e demonstração na aprendizagem razões trigonométricas

Recibido: mayo de 2013

Aceptado: agosto de 2013
Danny L. Algarín ${ }^{2}$

Jorge Enrique Fiallo Leal ${ }^{3}$

\begin{abstract}
Resumen
Presentamos avances de una investigación cuyo objetivo es caracterizar los niveles de razonamiento de Van Hiele específicos a los procesos de descripción, definición y demostración, entendidos como actividades cognitivas relacionadas con la comprensión y uso de los conocimientos en el tema de las razones trigonométricas. En una primera etapa elaboramos una caracterización a-priori de los procesos mencionados para cada uno de los niveles y diseñamos una unidad de enseñanza de las razones trigonométricas en un entorno de geometría dinámica, tendiente al aprendizaje de conceptos y de los procesos en los estudiantes. En esta comunicación, presentamos resultados parciales de la evolución de los estudiantes en el aprendizaje de los conceptos y procesos y un primer análisis a posteriori de la caracterización.
\end{abstract}

Palabras clave: Niveles de Van Hiele; descripción; definición; demostración; Matemáticas Escolares; Geometría, Trigonometría.

\begin{abstract}
We present progress of an investigation whose objective is to characterize the levels of van Hiele reasoning specific to the processes of description, definition and demonstration, understood as cognitive activities related to the understanding and use of knowledge on the subject of the trigonometric ratios. In a first step we developed a priori characterization of the processes mentioned for each of the levels and design a teaching unit of the trigonometric ratios in a dynamic geometry, aimed at learning of concepts and processes in students. In this paper, we present partial results of the evolution of the students in learning the concepts and processes and a first posteriori analysis of the characterization.
\end{abstract}


Keywords: Van Hiele levels, description, definition, demonstration, Mathematics Education, Geometry, Trigonometry.

\section{Resumo}

Apresentamos o progresso de uma investigação cujo objetivo é caracterizar os níveis de van Hiele raciocínio específicas para os processos de descrição, definição e demonstração, entendidas como atividades cognitivas relacionadas com a compreensão e uso do conhecimento sobre o assunto das razões trigonométricas. Num primeiro passo, nós desenvolvemos uma caracterização a priori dos processos mencionados para cada um dos níveis e conceber uma unidade de ensino das relações trigonométricas em uma geometria dinâmica, voltada para aprendizagem de conceitos e os processos em estudantes. Neste artigo, apresentamos os resultados parciais da evolução dos alunos em aprender os conceitos e processos e uma primeira análise a posteriori da caracterização.

Palavras-chave: Níveis de Van Hiele, descrição, definição, demonstração, educação matemática, geometria, trigonometria.

\section{Introducción}

Los alumnos llegan a los niveles superiores con muchas falencias en las competencias matemáticas, debido a los numerosos obstáculos y dificultades que se presentan para que el proceso de adquisición de competencias pueda ser exitoso. Una de las dificultades a las que se enfrentan docentes y estudiantes es la enseñanza y aprendizaje de la trigonometría.

Diversos estudios han contribuido al análisis de las dificultades en trigonometría, sin embargo tanto a nivel nacional (MEN, 1998; MEN, 2003, 2006) como a nivel internacional (NCTM, 2003) no se profundiza en el tema ni se presentan propuestas para afrontar el problema, lo cual ha llevado a que la trigonometría se enseñe de la misma forma (lo que está en los libros de texto) durante los últimos años. Tomando en cuenta lo anterior y con miras a lograr que el estudiante, a partir de situaciones concretas desarrolle su proceso de razonamiento, que adquiera y comprenda conceptos y relaciones matemáticas que favorezcan el aprendizaje de las razones trigonométricas, y sobre todo, que las situaciones planteadas favorezcan el tránsito de los estudiantes de un nivel de razonamiento a otro superior, nos formulamos la siguiente pregunta de investigación: ¿Cuáles son los descriptores que caracterizan los procesos de descripción, definición y demostración en cada uno de los niveles de razonamiento de Van Hiele de los estudiantes cuando se estudian las razones trigonométricas? Esta pregunta nos lleva al objetivo general: Caracterizar los niveles de razonamiento de Van Hiele específicos a los procesos de descripción, definición y demostración en el tema de las razones trigonométricas.

\section{Marco conceptual}

\section{Procesos matemáticos}

En la actividad matemática desarrollada en el aula de clase, intervienen procesos que se articulan en la medida que los estudiantes interactúan con las diversas situaciones planteadas. Estos procesos se entienden como actividades cognitivas relacionadas con la comprensión y el uso de los conocimientos.

Respecto a estos procesos, tenemos que: la palabra describir en todos los niveles de razonamiento puede asociarse a listas de propiedades o características de los conceptos (Guillén, 2004); la definición se concibe generalmente como un enunciado de las características y propiedades inherentes de un objeto matemático, las definiciones expresan las propiedades que los caracterizan (objetos) y los ubican dentro de una red de relaciones establecidas (Flórez, s.f.); la demostración se concibe como el proceso que incluye todos los argumentos 
planteados por los estudiantes para explicar, verificar, justificar o validar con miras a convencerse a sí mismo, a otros estudiantes y al profesor de la veracidad de una afirmación matemática (Fiallo, 2010)

\section{Modelo de Van Hiele}

El modelo de Van Hiele, está formado por dos componentes: los niveles de razonamiento, que describen la forma como los estudiantes razonan cuando efectúan diversas actividades para un tema, desde el razonamiento intuitivo hasta el razonamiento abstracto formal y las fases de aprendizaje, que ayudan al profesor a organizar las actividades para que sus estudiantes puedan avanzar de un nivel de razonamiento al inmediatamente superior.

\section{Niveles de Razonamiento}

El modelo considera cinco niveles de razonamiento, siendo el último nivel el de rigor, el cual no se alcanza en la escuela secundaria, por tanto, no lo tendremos en cuenta. Es característico del modelo el seguimiento de un orden, la adyacencia, las relaciones y el lenguaje propio de cada uno de los niveles, además, el paso de un nivel de pensamiento y conocimiento a otro no va asociado a la edad y sólo alcanzado un nivel se puede pasar al siguiente.

Nivel 1 Reconocimiento: los estudiantes razonan sobre conceptos básicos, tales como formas simples, principalmente por medio de consideraciones visuales del concepto como un todo (Burger \& Shaughnessy, 1986). Describen las propiedades y elementos físicos de los objetos matemáticos, no hay razonamiento matemático, por lo que no realizan ningún tipo de demostración (Gutiérrez, 2007).

Nivel 2 Análisis: los estudiantes razonan sobre los conceptos por medio de un análisis informal de las relaciones y propiedades, se establecen las propiedades necesarias del concepto (Burger \& Shaughnessy, 1986). Describen propiedades y elementos matemáticos de los conceptos, usan definiciones de estructura lógica simple, construyen definiciones a partir de un listado de las propiedades conocidas (Gutiérrez, 2007), realizan demostraciones de tipo empírico ingenuo, experimento crucial basado en ejemplo, experimento crucial constructivo y ejemplo genérico analítico (Fiallo, 2010)

Nivel 3 Deducción informal: los estudiantes ordenan lógicamente las propiedades de los conceptos, construyen definiciones abstractas y pueden distinguir entre la necesidad y suficiencia de un conjunto de propiedades al determinar un concepto (Burger \& Shaughnessy, 1986). Usan cualquier tipo de definición (Gutiérrez, 2007). Realizan demostraciones de tipo ejemplo genérico intelectual, experimento mental transformativo y experimento mental estructurado (Fiallo, 2010)

Nivel 4 Deducción formal: El estudiante razona formalmente dentro del contexto de un sistema matemático completo, con términos indefinidos, axiomas, un sistema lógico subyacente, definiciones y teoremas (Burger \& Shaughnessy, 1986). Admite la existencia de definiciones equivalentes, puede demostrar la equivalencia de definiciones (Gutiérrez, 2007). Realizan demostraciones: deductiva formal transformativa y deductiva formal estructurada (Fiallo, 2010).

\section{Fases de aprendizaje}

Según Crowley (1987), las características de las fases de aprendizaje son:

Fase 1 Información: En esta etapa inicial el profesor y los estudiantes conversan y realizan actividades sobre los objetos de estudio de este nivel.

Fase 2 Orientación dirigida: Los estudiantes exploran el tema de estudio a través de los materiales que el profesor ha ordenado cuidadosamente. Estas actividades deben revelar gradualmente a los estudiantes las estructuras características de este nivel.

Fase 3 Explicación: Apoyándose en sus experiencias previas, los estudiantes expresan e intercambian sus incipientes puntos de vista acerca de las estructuras que han observado.

Fase 4 Orientación libre: Los estudiantes encuentran tareas más complejas, tareas con muchos pasos, tareas que se pueden realizar de varias formas y actividades abiertas. 
Fase 5 Integración: Los estudiantes analizan y resumen lo que han aprendido, con el fin de tener una visión global de la nueva red de objetos y relaciones

\section{Unidad de enseñanza de las razones trigo- nométricas en un entorno de geometría dinámica.}

Se plantea una unidad de enseñanza conformada por 4 actividades: Razones trigonométricas para triángulos rectángulos, Razones trigonométricas para ángulos en posición normal, Representaciones lineales y visualización de las razones trigonométricas e Identidades Pitagóricas. Tiene como característica una enseñanza basada en el descubrimiento guiado basado en el uso de software de geometría dinámica. A partir de las interacciones con el software, los estudiantes pueden hacer descripciones de lo que observan en la pantalla, realizan generalizaciones que los llevan a usar y comprender definiciones, con las cuales elaboran demostraciones cada vez más formales. Se cuenta con archivos dinámicos, que se enfocan como herramienta de visualización, exploración y análisis de relaciones y propiedades trigonométricas.

\section{Metodologia}

La investigación, de tipo cualitativo, se está llevando a cabo con 35 estudiantes de $10^{\circ}$ grado ( $14-16$ años), de la Institución Educativa Luis Carlos Galán de Bucaramanga. La implementación de la unidad de enseñanza se realiza durante el horario normal de clases en el aula de informática, en grupos de dos estudiantes por computador. La recolección de los datos se hace a través de video grabación a dos grupos y lo escrito en las hojas de trabajo. A partir de las actuaciones de los estudiantes en la interacción con el computador, con los compañeros y con el investigador, y lo escrito, se obtienen datos cualitativos que son analizados para construir la caracterización de los descriptores de los niveles de razonamiento. La investigadora es a la vez la docente y se encarga de orientar las actividades a todo el curso, con la colaboración de un auxiliar (estudiante de Licenciatura en Matemáticas de la Universidad Industrial de Santander) quien conoce los propósitos de la investigación.

\section{Avances}

Hasta el momento se ha experimentado con la primera actividad, de cual han surgido actuaciones que han permitido afinar la caracterización inicial propuesta para cada uno de los procesos. Presentamos a continuación, algunos ejemplos con los cuales mostramos interacciones que nos permitieron afinar la caracterización inicial:

Ejemplo 1: Abre el archivo ACT 1, elabora una descripción de lo que observas en este archivo y halla las razones entre los lados del triángulo $\mathrm{ABC}$. Nombra cada razón con su respectivo cociente entre los lados del triángulo, por ejemplo $\mathrm{BC} / \mathrm{AB} .1$

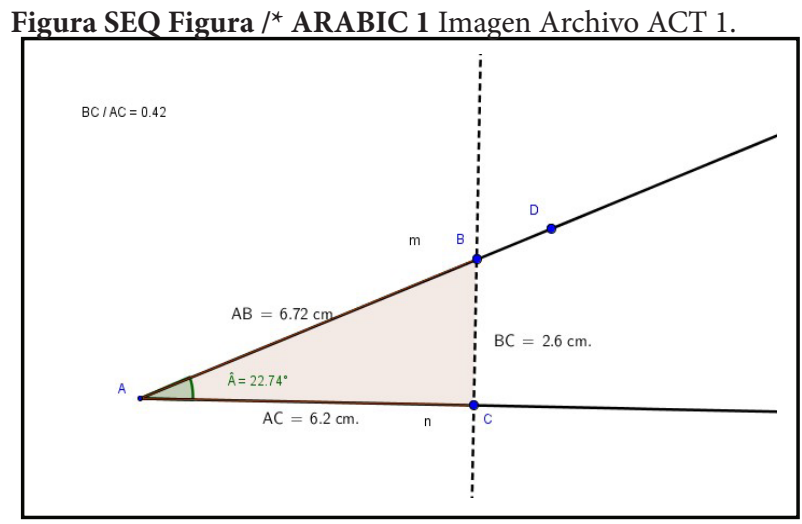

Fuente: Elaboración propia

Figura 2 Solución del estudiante

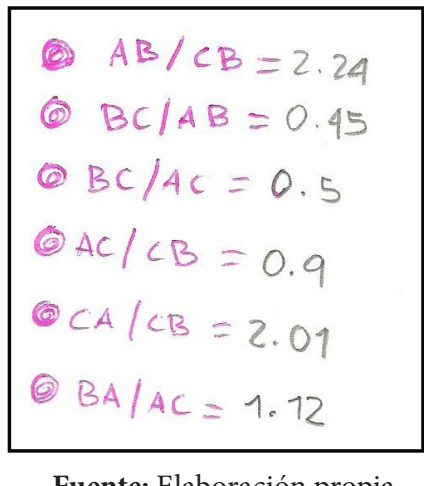

Fuente: Elaboración propia

Análisis: En este ejemplo observamos que el estudiante escribe valores para las razones trigonométricas, hace una descripción estática de estas, a pesar de observar cambios al arrastrar los puntos D y C. La descripción corresponde al nivel 2. 
Ejemplo 2: ¿Qué sucede con los valores de las razones cuando varía el ángulo entre $0^{\circ}$ y $90^{\circ}$ ? Escribe en tu hoja de trabajo una conjetura de lo encontrado. Describe todo lo que pensaste e hiciste para el planteamiento de tu conjetura. Explica por qué es verdadera tu conjetura.

Figura 3 Conjetura y demostración de un estudiante AB/CB=Podemos ver que lo rizón AB/CBicuand elaingub estaj en o sumedida quiere decir que disminuye

$A B / C B=P$ Pdthos ver que cuanda mobemos el punto D hacia aryiba la longitud de los

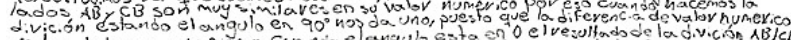

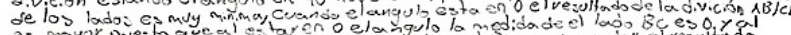

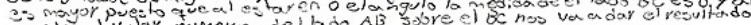

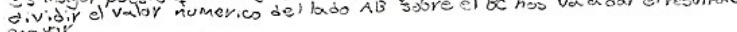

Fuente: Elaboración propia

Análisis: La demostración realizada por el estudiante es un Experimento Crucial Basado en Ejemplo, dado que el estudiante sólo considera los casos extremos $\left(0^{\circ}\right.$ y $\left.90^{\circ}\right)$, sin tener en cuenta lo que ocurre entre los valores correspondientes entre estos dos valores. Esta demostración corresponde a un experimento crucial y al nivel 2 .

\section{Conclusiones}

Los procedimientos desarrollados mediante la implementación de la primera actividad, permitieron a los estudiantes avanzar en sus niveles de razonamiento. El análisis cualitativo de los datos permitió establecer categorías emergentes para la descripción inicial propuesta de los niveles de Van Hiele con lo cual se ha ido perfeccionando la caracterización. Las discusiones plenarias favorecieron la comprensión de conceptos y propiedades que los estudiantes descubrieron por medio de la utilización del software, en esto se ha visto progreso en el avance lo numérico hacia lo algebraico y en la elaboración de demostraciones más formales.

\section{Referencias}

Fiallo, J. (2010). Estudio del proceso de Demostración en el aprendizaje de las Razones Trigonométricas en un ambiente de Geometría Dinámica. Universidad de Valencia, España.

Gutiérrez, A. (2007). Procesos matemáticos en la enseñanza/aprendizaje de la geometría. XVI Congreso Nacional de Matemáticas. Medellín.

Van Hiele, P.M. (1957). El problema de la comprensión (en conexión con la comprensión de los escolares en el aprendizaje de la geometría. Universidad de Utrecht, Utrecht (Traducción al español para el proyecto de investigación Gutiérrez y otros, 1991) 